\title{
$\checkmark$ A Patient with Encephalopathy Following Coil Embolization of Cerebral Aneurysm and Extracranial Stent Placement
}

Yoshiko Sagara, ${ }^{1}$ Hiro Kiyosue, ${ }^{2}$ Mika Okahara, ${ }^{3}$ Masatoshi Oga, ${ }^{1}$ Akihiko Kaga, ${ }^{4}$ and Yosuke Kamenofuchi ${ }^{4}$

Objective: We report a patient in whom encephalopathy developed after coil embolization of an unruptured basilar artery aneurysm and stent placement for vertebral artery stenosis.

Case Presentation: A 69-year-old female. When the unruptured basilar artery aneurysm was treated with coil embolization, a balloon-expandable stent was placed for left vertebral artery stenosis, and treatment was completed without complication. Loss of appetite and lightheadedness developed from 2 weeks after discharge, and multiple FLAIR highintensity areas, and nodular contrast enhancement in the left vertebral artery territory were observed on MRI. Steroid pulse therapy was performed suspecting metal allergy and foreign body granuloma, and symptoms improved.

Conclusion: Encephalopathy associated with foreign body granuloma and metal allergy may be caused by coil and stent placement. Patients should be sufficiently interviewed, and when allergies are suspected, reconsideration of the treatment method may be necessary in advance.

Keywords metal allergy, encephalopathy, complication, percutaneous transluminal angioplasty, extracranial stent

\section{Introduction}

Encephalopathy may develop as a complication of coil embolization, and various factors are considered with regard to the cause. We encountered a patient in whom stent placement was performed for extracranial vertebral artery stenosis subsequently to coil embolization of an unruptured basilar artery aneurysm, and many delayed abnormal intensity areas appeared in the brain parenchyma after treatment. Various causes including metal allergy were considered. We report the case with a literature review.

$\overline{{ }^{1} \text { Department of Radiology, Oitaken Koseiren, Tsurumi Hospital, }}$ Beppu, Oita, Japan

${ }^{2}$ Department of Radiology, Oita University Hospital, Yufu, Oita, Japan

${ }^{3}$ Department of Radiology, Shinbeppu Hospital, Beppu, Oita, Japan ${ }^{4}$ Department of Neurosurgery, Oitaken Koseiren, Tsurumi Hospital, Beppu, Oita, Japan

Received: June 5, 2018; Accepted: December 27, 2018 Corresponding author: Yoshiko Sagara. Department of Radiology, Oitaken Koseiren, Tsurumi Hospital, 4333 Tsurumi, Beppu, Oita 874-8585, Japan

Email: ysagara@oita-u.ac.jp



This work is licensed under a Creative Commons Attribution-NonCommercialNoDerivatives International License.

C2019 The Japanese Society for Neuroendovascular Therapy

\section{Case Presentation}

The patient was a 69-year-old female with hypertension being treated at the outpatient clinic. A right posterior inferior cerebellar artery aneurysm (diameter: $3.5 \times 3.7 \times$ $5.0 \mathrm{~mm}$ ) ruptured and caused subarachnoid hemorrhage (Hunt and Kosnik grade 3). The ruptured aneurysm was treated with embolization with two coils under general anesthesia (Fig. 1). No perioperative complication developed and the patient was discharged to home by independent walking on day 20. Treatment of an unruptured basilar artery aneurysm with coil embolization after 8 months was decided on. Since about $50 \%-60 \%$ stenosis was confirmed at the origin of the bilateral vertebral arteries in the previous treatment, concomitant percutaneous angioplasty was planned for stenosis of the left vertebral artery to use it as an access route. First, the basilar artery aneurysm was treated with stent-assisted intra-aneurysm coil embolization (8 coils $/ 60 \mathrm{~cm}$, volume embolization ratio: $35.4 \%$ ) (Fig. 2), and a balloon-expandable stent was placed in the left vertebral artery. Favorable dilatation could be acquired (Fig. 3) and treatment was completed with no intraoperative complication.

System used

Basilar artery aneurysm; 4Fr. FUBUKI guiding sheath (Asahi Intecc, Aichi, Japan), Headway 17 (MicroVention 


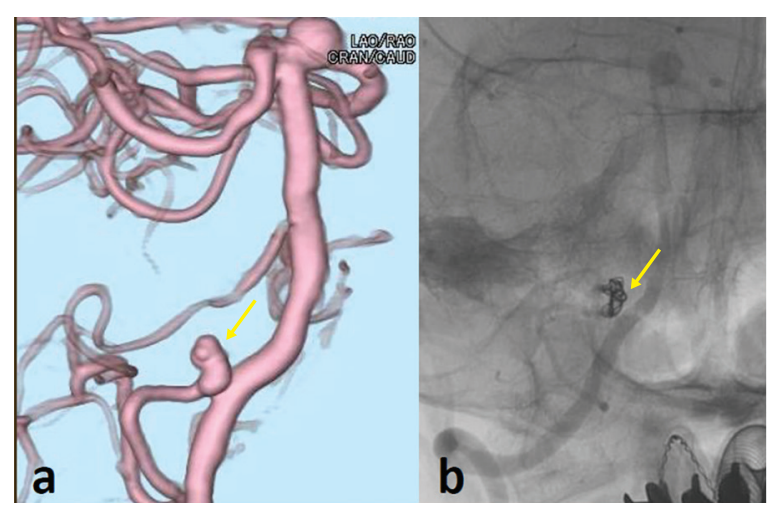

Fig. 1 (a) Reconstructed 3D image from rotation DSA before coil embolization, (b) angiography of the right vertebral artery, after coil placement in ruptured right posterior inferior cerebellar artery aneurysm (arrow). An unruptured basilar artery aneurysm is noted.

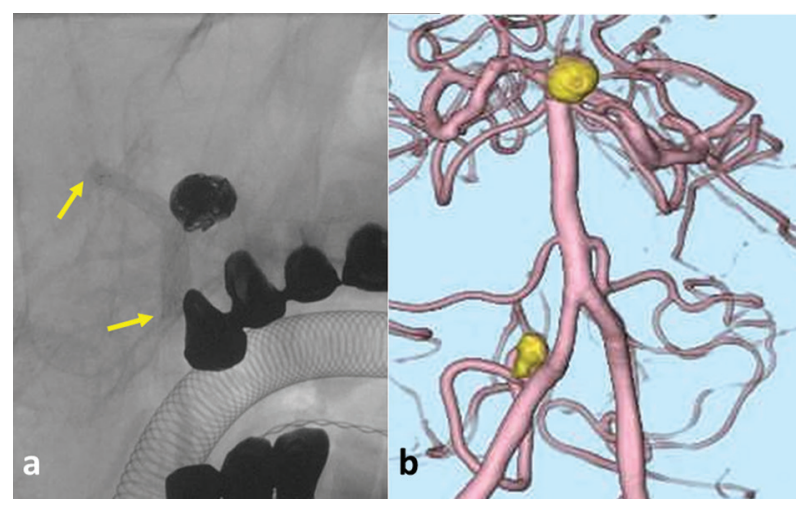

Fig. 2 (a) Intraoperative angiography of the vertebral artery, (b) Reconstructed 3D image from rotation DSA of the right vertebral artery after coil embolization. After stent (arrow)assisted intra-aneurysm coil embolization of the basilar artery aneurysm.



Fig. 3 (a) Angiography of the left subclavian artery shows about $50 \%$ stenosis at the origin of the left vertebral artery, (b) GuardWire (Medtronic, Minneapolis, MN, USA) is placed in the left distal vertebral artery for distal protection, (c) Express SD (Boston Scientific, Natick MA, USA) $4 \mathrm{~mm} / 15 \mathrm{mmL}$ was expanded at $8 \mathrm{~atm}$ (nominal $10 \mathrm{~atm}$ ) in the stenosis region, and favorable dilatation is acquired.

TERUMO, Tustin, CA, USA), Excelsior SL-10 (Stryker, Kalamazoo, MI, USA), CHIKAI 14 (Asahi Intecc), LVIS Jr (MicroVention TERUMO) $3.5 \mathrm{~mm} / 18 \mathrm{~mm}$, Target XL 360 soft (Stryker), $5 \mathrm{~mm} / 15 \mathrm{~cm}, 360 \mathrm{soft} 4 \mathrm{~mm} / 8 \mathrm{~cm}, 3.5 \mathrm{~mm} /$ $10 \mathrm{~cm}$, ultra $3.5 \mathrm{~mm} / 8 \mathrm{~cm}, 3.0 \mathrm{~mm} / 6 \mathrm{~cm}$, Nano $3.0 \mathrm{~mm} / 6 \mathrm{~cm}$, Axium PRIME (Covidien, Irvine, CA, USA), $2.5 \mathrm{~mm} /$ $4 \mathrm{~cm}$, ED COIL ExtraSoft (Kaneka Medix, Osaka, Japan), $2.0 \mathrm{~mm} / 3 \mathrm{~cm}$.

Left vertebral artery stenosis; under protection with GuardWire (Medtronic, Minneapolis, MN, USA), Express SD (Boston Scientific, Natick MA, USA) $4 \mathrm{~mm} / 15 \mathrm{mmL}$ was expanded at $8 \mathrm{~atm}$ (nominal $10 \mathrm{~atm}$ ).

At about 2 weeks after discharge, loss of appetite and lightheadedness developed and asthmatic symptoms appeared. On MRI, multiple patchy high-intensity areas appeared in the left medulla oblongata, bilateral cerebellar hemispheres, and occipital lobe on FLAIR (Fig. 4), and nodular enhancement was detected on Gd-enhanced imaging (Fig. 5). Since multiple lesions were noted in not only the bilateral posterior cerebral artery territory but also left vertebral artery territory, foreign body-induced granulomatous reaction and allergy to the stent placed in the left vertebral artery or instruments used in the procedure and contrast medium-induced encephalopathy were considered. The cell count and protein level in cerebrospinal fluid slightly increased, but culture was negative. On blood testing, slight increases in the white blood cell count and IgE level $(563 \mathrm{U} / \mathrm{mL})$ were noted, but the inflammatory reaction was not elevated. The patient was re-interviewed and it was clarified that contact dermatitis previously developed in response to a metal belt of a watch, suggesting that metal allergy-associated encephalopathy is one possibility. The 


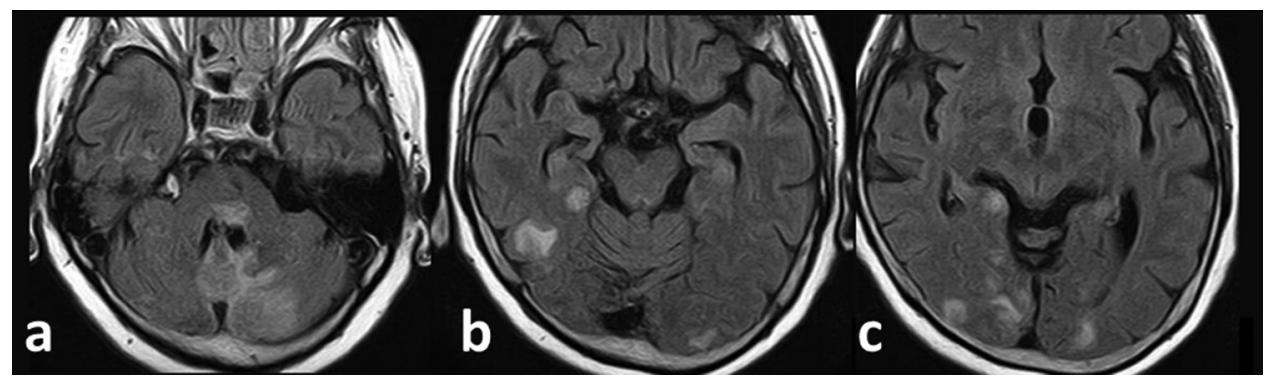

Fig. 4 (a-c) MRI FLAIR images 1 month after the second treatment show multiple abnormal high-intensity areas in the brain stem, left cerebellar hemisphere, and bilateral occipital lobes.

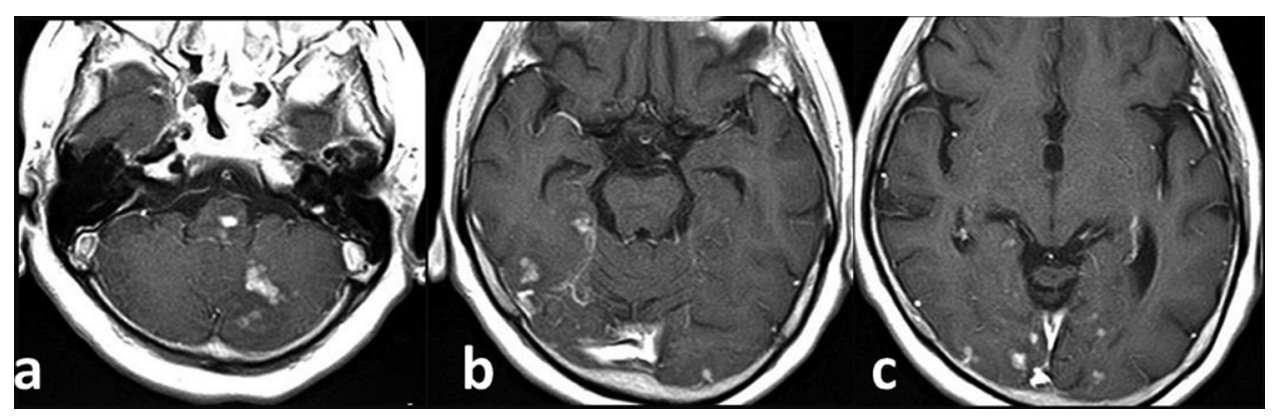

Fig. 5 (a-c) MRI Gd-enhanced T1-weighted images 1 month after the second treatment. Gd-enhancement is noted in regions consistent with the high-intensity areas on FLAIR in the brain stem, left cerebellar hemisphere, and bilateral occipital lobes.

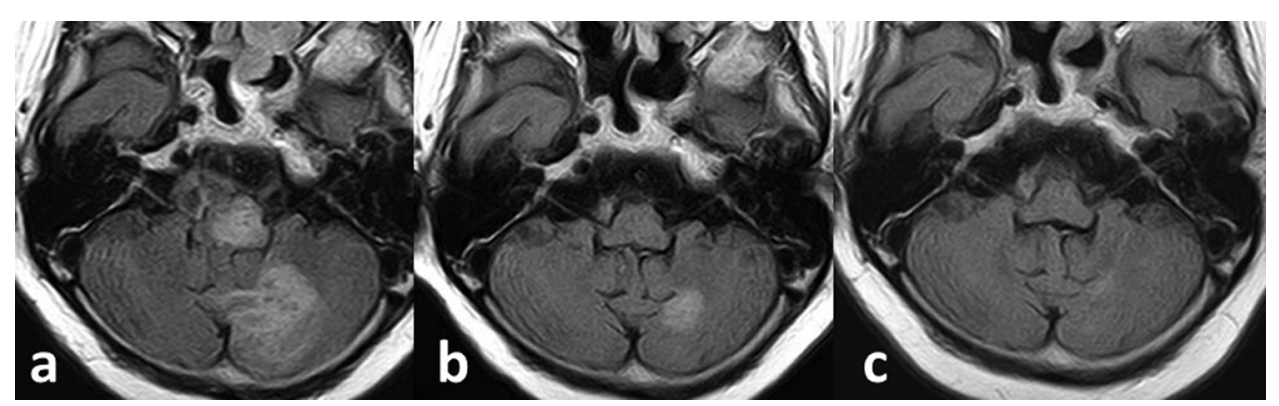

Fig. 6 Time-course changes in MRI FLAIR images. (a) After 1 month, (b) after 2.5 months, and (c) after 4 months. The abnormal intensity areas are mostly resolved by steroid treatment.

symptoms were remitted by steroid pulse therapy (methylprednisolone $1000 \mathrm{mg}$ for 3 days) and steroid tapering was subsequently performed (dose reduction from prednisolone $30 \mathrm{mg}$ /day). The lesions mostly disappeared within 3.5 months after initiation of steroid therapy on MRI (Fig. 6), and the patient is currently being followed at the outpatient clinic.

\section{Discussion}

With the recent spread of coil embolization of cerebral aneurysms, multiple enhanced lesions in the brain following coil embolization have been occasionally reported, and vasculitis, contrast medium-induced encephalopathy, foreign body granuloma induced by iatrogenic embolism with hydrophilic coating detached from bioactive coils and catheters, and coil-associated delayed metal allergy have been discussed as the causes. ${ }^{1-9)}$ Moreover, metal embolism was suspected in a case although it was asymptomatic. ${ }^{10)}$

Regarding foreign body granuloma induced by iatrogenic embolism with hydrophilic coating materials, granuloma has been identified by histopathological examination in some cases. Shapiro et al. reported that the foreign body was polyvinylpyrrolidone (PVP) coating of a catheter. ${ }^{\text {) }}$

In our patient, multiple lesions were present in not only the bilateral posterior cerebral artery territory but also other regions including the left vertebral artery territory; therefore, embolism and foreign body granuloma caused by 
eluted metal ions and detached foreign bodies from the stent placed in the left extracranial vertebral artery or devices placed in the left vertebral artery during treatment, allergy, and contrast medium-induced encephalopathy were considered. However, no abnormality was detected on angiography after the first treatment of the ruptured aneurysm or later angiography, suggesting that contrast mediuminduced encephalopathy is unlikely. Platinum allergy to coils is also unlikely because encephalopathy did not develop following the first treatment of the ruptured aneurysm and the distribution of the lesions was not consistent with the region with coil placement. Regarding embolism with detached hydrophilic coating materials, tight-fitting coaxial catheter systems and friction among catheters due to the use of several inner catheters are included in the causes ${ }^{7)}$ and FDA called attention to these. Oh et al. ${ }^{11)}$ reported that bending of the microcatheter during coil insertion and repeated coil insertion/removal caused detachment of the inner coating on benchtop analysis. It is described in the package inserts that hydrophilic coating was applied to all the guiding catheters, microcatheters, and guidewires used for embolization in our patient, and the same guiding catheter, guidewire, and microcatheter (Headway 17) were used in the first and second treatments. In the second treatment, Excelsior SL-10 was additionally used as the second microcatheter, the use of Express SD and GuardWire, and the type of coils were different. Two microcatheters were inserted in the guiding catheter in the second treatment, but no problem, such as interference with the catheter and unraveling of the coils, occurred during the procedure. No event suggesting damage of the devices occurred during treatment, and no finding suggesting the presence of a foreign body was detected in the susceptibility imaging on MRI performed immediately after treatment. If hydrophilic coating was detached from the inner wall of microcatheter, lesions would have been distributed in the posterior cerebral artery territory. However, since the guiding catheter was placed in the vertebral artery, it cannot be ruled out that embolus produced by the detachment of coating from the guiding catheter and guidewire were scattered in the vertebral artery territory.

A definite diagnosis was not made because steroid pulse therapy was promptly initiated and allergic reaction on a patch test was not confirmed, but the symptoms manifested 3 weeks after treatment, suggesting that delayed type IV allergy to the foreign body and metal was a cause.

The association between re-stenosis and metal allergy stent placement for renal arterial stenosis and coronary arterial stenosis has been reported. ${ }^{12)}$ In type III Kounis syndrome in which allergic reaction and acute coronary syndrome simultaneously develop, embolism occurs in the coronary arterial stent and biological allergic reaction is considered the main pathology. ${ }^{13)}$ Allergic reaction to an occluder (nitinol wire) percutaneously placed to close patent foramen ovale has also been reported. ${ }^{14-16)}$

We could not find a study reporting development of an intracranial lesion as a complication of stent placement in the extracranial vertebral artery or subclavian artery in literature search, but four cases of multiple cerebral lesions considered nickel allergy to coils placed to embolize intracranial aneurysms have been reported ${ }^{17-19)}$ and stentassisted embolization had been performed in three of them. A patch test was performed in two and allergic reaction to nickel was confirmed. Park et al. reported two cases of multiple white matter lesions which developed 18 and 21 days after stent-assisted (Enterprise VRD: Codman, Miami, FL, USA) coil embolization of unruptured cerebral aneurysm, and a patch test including platinum was performed in one patient and only nickel was positive. ${ }^{17)}$ In our patient, although the composition of the metal belt to which she reacted could not be confirmed, nickel is frequently an allergen among metals at a frequency of $7 \%-15 \%$ in the general population, and reportedly, $16.2 \%$ of patients with contact dermatitis are hypersensitive to nickel. Eluted metal ions bind to protein and become allergens, and serum nickel ions promote inflammatory reactions of the surrounding tissue by activating IL-1 $\beta$, cell adhesion molecule, and monocytes. ${ }^{17)}$ About $12 \%$ nickel is contained in $316 \mathrm{~L}$ stainless steel used in coronary stents and peripheral vascular stents, and renal artery stents such as Express SD placed in the vertebral artery in our patient. Moreover, nitinol constituting intracranial stents, such as LVIS Jr used for stent-assisted intra-aneurysm coil embolization, is an alloy comprised of $55 \%$ nickel and 45\% titanium. Therefore, when a nickelcontaining device is used in a patient with metal allergy, the possibility of developing allergic inflammatory reaction cannot be ruled out. In a study in which the serum nickel level was measured after insertion of a nitinol-containing device, the level started to rise 24 hours after surgery, peaked at 1 month, and returned to the standard level after 12 months. ${ }^{16)}$ This time course may be consistent with the course of encephalopathy in our patient and other cases of nickel allergy-induced encephalopathy.

It has been reported by Köster et al. in Lancet 2000 that re-stenosis may be enhanced by metal allergy to nickel and molybdenum eluted from stainless coronary arterial stents, ${ }^{12}$ ) and subsequently, revision of descriptions based on the 
Pharmaceutical Affairs Law was instructed. It is described in the package inserts of various types of stent that "eluted metals may induce metal allergy and it is necessary to reinvestigate the validity of stent treatment for patients with metal allergy." Although the influence of allergy to stents for intracranial cerebral aneurysm embolization and arteriosclerosis cannot be evaluated based on the data of coronary arterial stents, ${ }^{20)}$ patients should be sufficiently interviewed before treatment for either case. Unfortunately, the cause of encephalopathy was not identified in our patient. Various causes were considered including metal allergy as one possibility. When the presence of metal allergy is clarified before treatment, the indication should be verified or a patch test should be performed, and pretreatment to reduce adverse effects and perioperative management should be performed. It is also necessary to sufficiently explain the possibility of foreign body granuloma formation as a complication to patients before surgery and pay attention to the selection and operation of catheters to prevent foreign body detachment from the catheters.

\section{Conclusion}

Coil and stent placements may cause foreign body granuloma and metal allergy-associated encephalopathy. Patients should be sufficiently interviewed, and when allergy is suspected, reconsideration of the treatment method may be necessary.

\section{Disclosure Statement}

None of the first and co-authors have conflict of interest.

\section{References}

1) Dues-Silva L, Lum C, De Meulemeester C, et al: Severe aggressive acute disseminated encephalomyelitis-like reaction after aneurysm coiling. Neurosurgery 2010; 66: 222-223.

2) Uchiyama $Y$, Abe T, Hirohata M, et al: Blood brain-barrier disruption of nonionic iodinated contrast medium following coil embolization of a ruptured intracerebral aneurysm. AJNR Am J Neuroradiol 2004; 25: 1783-1786.

3) Mutoh T, Ishikawa T, Sawada M, et al: [Cerebral complications induced by neurotoxity of nonionic constant medium after embolization of unruptured cerebral aneurysms: report of 2 cases]. No Shinkei Geka 2010; 38: 163-170. (in Japanese)

4) Skolarus LE, Gemmete JJ, Braley T, et al: Abnormal white matter changes after cerebral aneurysm treatment with polyglycolic-polylactic acid coils. World Neurosurg 2010; 74: 640-644.
5) Mehta RI, Mehta RI, Solis OE, et al: Hydrophilic polymer emboli: an under-recognized iatrogenic cause of ischemia and infarct. Mod Pathol 2010; 23: 921-930.

6) Cruz JP, Marotta T, O'Kelly C, et al: Enhancing brain lesions after endovascular treatment of aneurysms. AJNR Am J Neuroradiol 2014; 35: 1954-1958.

7) Shapiro M, Ollenschleger MD, Baccin C, et al: Foreign body emboli following cerebrovascular interventions: clinical, radiographic, and histopathologic features. AJNR Am J Neuroradiol 2015; 36: 2121-2126.

8) Mehta RI, Mehta RI: Polymer-induced central nervous system complications following vascular procedures: spectrum of iatrogenic injuries and review of outcomes. Hum Pathol 2016; 53: 178-190.

9) Okawara M, Son J, Yamaguchi H, et al: [A case of multiple enhancing lesions after coil embolization: the association with hydrophilic polymer emboli]. NKC 2016; 1: 37-43. (in Japanese)

10) Yasuda R, Maeda M, Umino M, et al: Suspected metallic embolism following endovascular treatment of intracranial aneurysms. AJNR Am J Neuroradiol 2016; 37: 1696-1699.

11) Oh SW, Shin NY, Lee HJ, et al: Delayed enhancing lesions after coil embolization of aneurysms: clinical experience and benchtop analyses. J Neurointerv Surg 2017; 9: 1243-1247.

12) Köster R, Vieluf D, Kiehn M, et al: Nickel and molybdenum contact allergies in patients with coronary in-stent restenosis. Lancet 2000; 356: 1895-1897.

13) Almpanis GC, Tsigkas GG, Koutsojannis C, et al: Nickel allergy, Kounis syndrome and intracardiac metal devices. Int J Cardiol 2010; 145: 364-365.

14) Fukahara K, Minami K, Reiss N, et al: Systemic allergic reaction to the percutaneous patent foramen ovale occluder. J Thorac Cardiovasc Surg 2003; 125: 213-214.

15) Dasika UK, Kanter KR, Vincent R: Nickel allergy to the percutaneous patent foramen ovale occluder and subsequent systemic nickel allergy. J Thorac Cardiovasc Surg 2003; 126: 2112.

16) Ries MW, Kampmann C, Rupprecht HJ, et al: Nickel release after implantation of the Amplatzer occluder. Am Heart J 2003; 145: 737-741.

17) Park HS, Nakagawa I, Yokoyama S, et al: Nickel-associated delayed multiple white matter lesions after stent-assisted coil embolization of intracranial unruptured aneurysm. BMJ Case Rep 2017; 2017: pii: bcr2017013005.

18) Ulus S, Yakupoğlu A, Kararslan E, et al: Reversible intracranial parenchymal changes in MRI after MCA aneurysm treatment with stent-assisted coiling technique; possible nickel allergy. Neuroradiology 2012; 54: 897-899.

19) Lobotesis K, Mahady K, Ganesalingam J, et al: Coilingassociated delayed cerebral hypersensitivity: Is nickel the link? Neurology 2015; 84: 97-99.

20) Jeswani S, Alexander MJ: Nickel allergy: a reason for concern? J Neurointerv Surg 2011; 3: 2-4. 